\title{
Combining Handhelds with a Whole-Class Display to Support the Learning of Scientific Control
}

\author{
Tom Moher ${ }^{1}$, Xun Ding ${ }^{1}$, Jennifer Wiley ${ }^{2}$, Diane Conmy ${ }^{3}$, Syeda Hussain ${ }^{1}$, \\ Preeti Singh $^{1}$, Vasisht Srinivasan ${ }^{1}$ \\ ${ }^{1}$ Department of Computer Science \\ ${ }^{2}$ Department of Psychology \\ University of Illinois at Chicago \\ Chicago, IL 60607 USA \\ moher@uic.edu \\ ${ }^{3}$ Abraham Lincoln Elementary School \\ Oak Park District 97 Public Schools \\ Oak Park, IL 60304 USA \\ dconmy@op97.org
}

\begin{abstract}
Third grade students used wireless handhelds and a large shared display to discover strategies for control of variables in scientific experiments. The technology suite supported activity requirements including synchronous individual control, face-to-face discourse, and instantaneous display updates. In an empirical study, students demonstrated learning in both original and transfer domains.
\end{abstract}

\section{Keywords}

Wireless handhelds, shared displays, classroom technologies, inquiry learning, scientific control

\section{INTRODUCTION}

The emergence of wireless handheld devices has had a stimulating effect on the educational technology research community because of their potential to support fundamentally new kinds of learning activities [5,7]. The limited screen size of handheld devices, however, makes their use problematic in whole-class instructional contexts that require a shared visual discourse referent. One alternative is to add a shared display to reflect the aggregate work conducted using individual handheld devices $[2,4,8]$.

In this paper we describe a learning application that combines networked wireless handhelds and a large shared display to help third-grade students gain experience with a fundamental concept of experimental science: the understanding that in a multivariate system only one variable should be manipulated at a time while others are held constant. This control of variables (COV) strategy is a relatively difficult concept; even college-aged students show unreliable application of the strategy across content domains [3,6]. Chen and Klahr [1] demonstrated that children in second through fourth grades could be relatively successful at adopting the COV strategy when they had both an opportunity to design their own experiments and explicit ("direct") instruction in designing unconfounded experiments.
Our goal was to extend this work by looking at the acquisition of the COV strategy in a discovery-learning context. We designed an activity, Who's Who?, in which each student simultaneously controls an independent variable. In order to promote discovery of the COV strategy, the activity requires that the manipulation of independent variables be instantaneous, and that all participants can see and discuss the effects of those manipulations. Our hypothesis was that universal involvement, external feedback from a large visual display, and the need to collaborate with peers would combine to lead to acquisition of the strategy without the need for direct instruction.

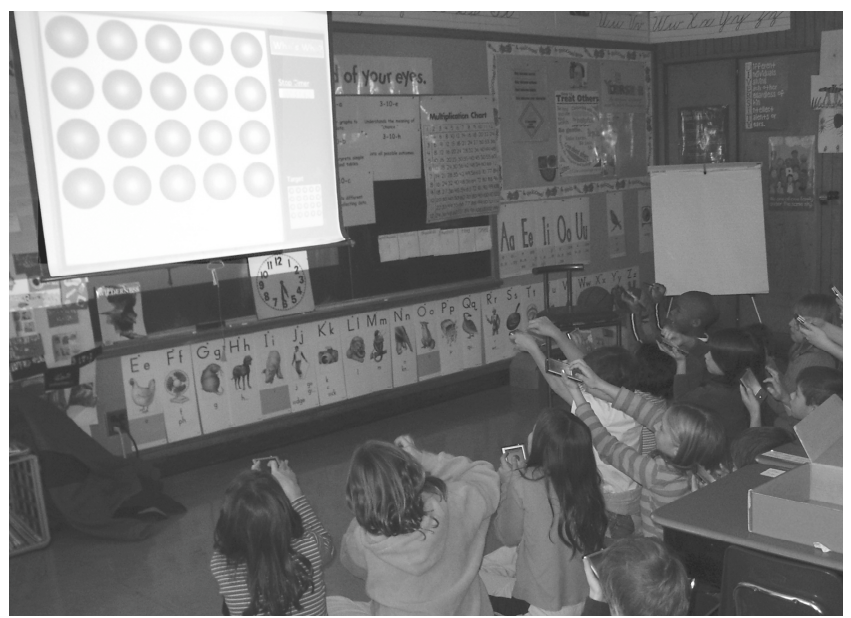

Figure 1. Third grade students using wireless PocketPCs to control "pixel" colors in Who's Who.

\section{WHO'S WHO?}

In Who's Who?, a group of users sits before a projected image consisting of a grid of large "pixels" (Fig. 1), randomly colored either blue or orange. Each user has a handheld PocketPC displaying a button; when the button is touched, an animated hand "presses" the button (depress and release), which then returns to its (single) stable state. Each handheld is mapped to a different pixel, and each button press toggles the color of that pixel; however, users are informed of neither the mapping nor the action of the buttons. The group is asked to modify the color of the individual pixels so that the final configuration matches that of a specified target pattern (in this case, all blue 
pixels) as quickly as possible. In order to complete the task, the collective group of users needs to discover (a) the mechanism by which pixel colors are changed (e.g., 1-to-1 mapping between handhelds and pixels, button toggles state), (b) a strategy for effecting the global state change (COV, i.e., one person at a time), and (c) a social process for implementing that strategy (e.g., centralized control directed by one student).

\section{METHOD}

Seventeen third-grade children, including eight girls and nine boys, completed all activities in the study. Each child was given a written test to evaluate his or her understanding of the COV strategy in an alternative domain (articulation of an effective strategy for assessing which of three ingredients in a mixture of dog food caused the dog to refuse to eat). The whole class played Who's Who on consecutive day, with different student-to-pixel mappings on each day. Each child was assessed using a structured interview after each session and, following independent review of videotape protocols, assigned a binary coding (understood/did not understand) of the task-specific mechanism and strategy task learning goals. (The social process analysis is not yet completed.) The canine diet preference was administered again following a three-week delay to test for $\mathrm{COV}$ concept retention and transfer.

\section{RESULTS}

\section{Task completion}

The children succeeded in reaching the goal configuration in both activity sessions; however, the contrast between the two was dramatic. In the first session, 32:47 minutes were required to complete the task. The second session lasted only 3:54 minutes.

\section{User activity}

Students averaged a remarkable 490 button presses apiece (range 147 to 939) during the first activity, or about one press per user every five seconds. During the second session, students averaged 11.7 button presses (range 1 to 31 ), or about one press every 20 seconds; four of the students pressed the button the minimum number of times (either once or twice, depending on the initial state of their pixel). Girls averaged 574 presses to the boys' 406 during the first session, but activity by gender during the second session was virtually identical.

\section{Learning}

Most of the task strategy learning occurred during the first session, with $94 \%$ of the students articulating a "one at a time" algorithm after each activity. Mechanism understanding increased from $65 \%$ to $88 \%$ between the two sessions. Conceptual learning, as indicated by the ability to apply the $\mathrm{COV}$ in the alternative domain, improved significantly from pre- to post-test $\left(X^{2}(1)=5.1, p<.03\right)$, with $53 \%$ students able to articulate an effective COV strategy prior to the Who's Who activities, and $88 \%$ afterward.

\section{DISCUSSION}

In this study, the combination of wireless handhelds and a shared display afforded a new activity structure that could not have been realized without technological support.
The reduction in task time demonstrates that the students learned how to apply the COV strategy in Who's Who? without the need for direct instruction at an "expert" level; their time on the second round was nearly identical to that of a pilot group of 16 adults. The process of coming to understand entailed the evaluation of a variety of theories regarding mechanism (e.g., that the pixels changed states randomly, that the handheld-to-pixel mapping was based on pointing or the ID numbers printed on the back of the handhelds) and strategy (e.g., that simultaneous pressing of the buttons would effect the desired state change) that were ultimately resolved by experimental procedures established through group discourse. Pre/post differences on the transfer test lend preliminary evidence of conceptual learning. Subsequent analyses will focus on social processes during discovery, using activity discourse data and the classroom teacher's characterization of established interpersonal relationships, in order to better understand how this technology configuration mediated learning and behavior within the whole-class context.

\section{ACKNOWLEDGMENTS}

Thanks to the UIC Electronic Visualization Laboratory, including Manuel Sanchez and Lance Long, for their help in videotaping and conducting post-activity interviews. This work was conducted with the support of NSF award EIA-9802090.

\section{REFERENCES}

1. Chen, Z. and Klahr, D. All other things being equal: Acquisition and transfer of the control of variables strategy. Child Development 70, 1098-1120.

2. Dufresne, R., Gerace, W., Leonard, W., Mestre, J., and Wenk, L. Classtalk: A classroom communication system for active learning. Journal of Computing in Higher Education 7, 3-47.

3. Kuhn, D., Amsel, E., and O'Laughlin, M. The Development of Scientific Thinking Skills. Academic Press, San Diego, CA, 1988.

4. Rekimoto, J. A multiple device approach for supporting whiteboard-based interactions, in Proceedings of $\mathrm{CHI}$ '98, ACM Press, 18-23.

5. Roschelle, J., Pea, R. A walk on the wild side: How wireless handhelds may change CSCL, in Proceedings of CSCL 2002 (Boulder, CO, Jan. 2002), Lawrence Erlbaum, Hillsdale NJ, 51-60.

6. Schauble, L. The development of scientific reasoning in knowledge-rich contexts. Developmental Psychology 32, 102-109.

7. Soloway, E., Grant, W., Tinker, R., Roschelle, J., Mills, M., Resnick, M., Berg, R. and Eisenberg, M. Science in the Palms of Their Hands. Commun. ACM $38,8,21-27$.

8. Wilensky, U., and Stroup, W. Networked Gridlock: Students Enacting Complex Dynamic Phenomena with the HubNet Architecture, in Proceedings of ICLS 2000 (Ann Arbor, MI, June 2000), Lawrence Erlbaum, Hillsdale NJ, 282-289. 